\title{
The reduced form of a game
}

Citation for published version (APA):

Vermeulen, D., \& Jansen, M. J. M. (1997). The reduced form of a game. METEOR, Maastricht University School of Business and Economics. METEOR Research Memorandum No. 001 https://doi.org/10.26481/umamet.1997001

Document status and date:

Published: 01/01/1997

DOI:

10.26481/umamet.1997001

Document Version:

Publisher's PDF, also known as Version of record

\section{Please check the document version of this publication:}

- A submitted manuscript is the version of the article upon submission and before peer-review. There can be important differences between the submitted version and the official published version of record.

People interested in the research are advised to contact the author for the final version of the publication, or visit the DOI to the publisher's website.

- The final author version and the galley proof are versions of the publication after peer review.

- The final published version features the final layout of the paper including the volume, issue and page numbers.

Link to publication

\footnotetext{
General rights rights.

- You may freely distribute the URL identifying the publication in the public portal. please follow below link for the End User Agreement:

www.umlib.nl/taverne-license

Take down policy

If you believe that this document breaches copyright please contact us at:

repository@maastrichtuniversity.nl

providing details and we will investigate your claim.
}

Copyright and moral rights for the publications made accessible in the public portal are retained by the authors and/or other copyright owners and it is a condition of accessing publications that users recognise and abide by the legal requirements associated with these

- Users may download and print one copy of any publication from the public portal for the purpose of private study or research.

- You may not further distribute the material or use it for any profit-making activity or commercial gain

If the publication is distributed under the terms of Article $25 \mathrm{fa}$ of the Dutch Copyright Act, indicated by the "Taverne" license above, 


\title{
THE REDUCED FORM OF A GAME
}

\author{
Dries Vermeulen and Mathijs Jansen
}

\begin{abstract}
The goal of this paper is twofold. Firstly a short proof of the unicity of the reduced form of a normal form game is provided, using a technique to reduce a game originally introduced by Mertens. Secondly a direct combinatorial-geometric interpretation of the reduced form is described. This description is then used to derive an algorithm for the calculation of the reduced form of a game.
\end{abstract}

\section{Introduction}

Usually the strategy space of the normal form of an extensive form game contains a number of 'duplicate' pure strategies. Such duplicate pure strategies arise when in the extensive form game a player has to specify his choices in a part of the decision tree that is not reached in the eventual play of the game (due to his own choices earlier in the tree). In the normal form of the game these duplicates lead to the same payoff for every player, no matter what the other players do. This specific property of duplicate strategies is usually referred to as payoff equivalence.

Before solving a game using the normal form, we are inclined to delete all but one of such payoffequivalent pure strategies from the normal form, since the resulting game is easier to handle while the strategic possibilities of the players are not changed. Thus also the eventual solution of the game should not be altered by this deletion process. The final result of such a deletion process is referred to as the semi-reduced normal form of the (extensive form) game. The unicity of this semi-reduced normal form up to changes in the names of the pure strategies is intuitively clear since the elimination process only involves the preservation of exactly one element of each collection of payoff-equivalent pure strategies.

Kohlberg and Mertens (1986) systematically investigated to what further extent payoff-equivalent strategies can be deleted from a normal form game. They argued that the process of deletion should 
not only concern the removal of duplicate pure strategies. In their opinion also pure strategies that are payoff equivalent to other (possibly mixed) strategies can be deleted from the normal form without harming the eventual solution. Thus they introduced the reduced normal form as the game 'where all pure strategies that are (payoff equivalent with) convex combinations of other pure strategies have been deleted'.

Although this description of the reduced normal form appeals to the intuition it also has some drawbacks. Unlike with the earlier definition of the semi-reduced normal form, it is in this case not immediately clear that the process will lead to a (in some sense) uniquely defined game. ${ }^{\dagger}$ The main problem being the fact that the deletion process is necessarily performed successively, one after the other. Thus, the resulting game depends on the order in which pure strategies are deleted and a game may have different reduced normal forms. It is even not immediately clear that two exhaustive sequences of deletions require the same number of deletions. People who have tried to construct a direct proof of the existence of a unique reduced normal form in some sense may have noticed that a rigorous proof can get quite involved indeed.

Nevertheless, it will be shown that all reduced normal forms of a normal form game are identical up to what is called 'the relabeling of pure strategies' in Mertens (1987). The proof is based on the technique used by Mertens in his mimeo for the identification of games. This technique is in fact an elegant way to capture the process of deletion in mathematical terms, as well as the 'relabeling' of pure strategies. Given this technique the proof becomes fairly straightforward and quite short.

The second problem with the description by Kohlberg and Mertens is that it is still not clear which game will eventually come out of the process of deletion of pure strategies, even if the existence of a unique reduced normal form of a normal form game is taken for granted.

Concerning this problem, most people automatically feel that, although two reduced normal forms of a game may be different on a formal level as discussed above, they should be very much alike, simply because it must be possible to predict on beforehand which pure strategies are going to be deleted from the original game in the above process. We will show that this is indeed the case. Given an arbitrary (normal form) game, the results of the first part are used to derive a direct combinatorial-geometric interpretation of the reduced normal form of that game. On one hand, this interpretation may serve as an alternative definition of the reduced normal form. On the other hand it accurately describes which pure strategies are superfluous and which are not. More precisely, exactly one pure strategy payoff equivalent with a given pure strategy remains in the reduced form if and only if the collection of strategies that are payoff equivalent with the given

\footnotetext{
$\dagger$ This problem was pointed out to us by an anonymous referee and the editor in charge of a
} previous paper. 
pure strategy is a face of the strategy space of the player involved. All other pure strategies vanish completely. Finally an algorithm based on this combinatorial-geometric interpretation is given for the actual computation of the reduced normal form.

\section{Contents of the paper}

Section 2 is, save the preliminaries, concerned with the relation between the deletion of a pure strategy of a given game, and the notion of reductions of,. In section 3 the unicity of the reduced form of, is proved using the language developed in section 2. At the end of section 3 a criterion is provided to check whether a given game actually is the reduced form of the game, . In section 4 this criterion is used to show that a specific game constructed directly from, equals its reduced form. In section 5 it is shown that this construction can be performed in finite time.

Notation For a finite set $T,|T|$ denotes the number of elements of $T$. For a convex set $C$, $\operatorname{ext}(C)$ denotes the set of extreme points of $C$ and for a set $D, \operatorname{ch}(D)$ denotes the convex hull of $D$.

\section{Reductions and deletion of pure strategies}

In this section we will establish the relation between the deletion of a pure strategy and the notion of a reduction of a game. (From now on we will omit the prefix 'normal form', since we will work exclusively in the normal form context.) The latter notion of a reduction is based on a technique introduced by Mertens (1987) and will in the next section turn out to be the ideal tool for stating precisely in what sense reduced forms are identical. In order to put our arguments for these assertions on a sound basis we first need some notation.

For a natural number $n, N:=\{1, \ldots, n\}$. An (n-person) game is a pair,$=\langle A, u\rangle$ such that $A:=\prod_{i} A_{i}$ is a product of $n$ non-empty, finite sets and $u=\left(u_{i}\right)_{i \in N}$ is an $n$-tuple of functions $u_{i}: A \rightarrow \mathbb{R}$. Here $A_{i}$ is the set of pure strategies of player $i$ and $u_{i}$ is his payoff function.

As usual, a game, will be identified with its mixed extension. For this game, the mixed strategies of player $i$ are the elements of the set $\Delta\left(A_{i}\right)$ of probability distributions on $A_{i}$. By abuse of notation we will identify a pure strategy $a \in A_{i}$ with the mixed strategy in $\Delta\left(A_{i}\right)$ that puts all weight on $a$. So, $A_{i}$ will simply be viewed as a subset of $\Delta\left(A_{i}\right)$. Also the pure strategy profiles will be denoted by $a \in A$. In case confusion might occur we will write $a_{i} \in A_{i}$ instead of simply $a \in A_{i}$.

For a (mixed) strategy profile $x=\left(x_{i}\right)_{i \in N} \in \Delta_{A}:=\prod_{j} \Delta\left(A_{j}\right)$, the (expected) payoff function of player $i$ is defined by $u_{i}(x):=\sum_{a \in A} \prod_{j} x_{j a_{j}} u_{i}(a)$. Two strategies $y_{j}$ and $z_{j}$ of player $j$ are called payoff equivalent if for all $i$ and all $x_{-j} \in \prod_{h \neq j} \Delta\left(A_{h}\right)$

$$
u_{i}\left(x_{-j} \mid y_{j}\right)=u_{i}\left(x_{-j} \mid z_{j}\right)
$$

In the displayed strategy profile $\left(x_{-j} \mid y_{j}\right) \in \Delta_{A}$ player $j$ uses the strategy $y_{j} \in \Delta\left(A_{j}\right)$ and his 
opponents use the strategies in $x_{-j} \in \prod_{i \neq j} \Delta\left(A_{i}\right)$.

Now let,$=\langle A, u\rangle$ be a game. In order to formalize the process of (successive) deletions as described by Kohlberg and Mertens, let $b \in A_{j}$ be a pure strategy that is payoff equivalent with some other (mixed) strategy $z_{j} \in \Delta\left(A_{j}\right)$. Then the game,${ }^{\prime}=\left\langle A^{\prime}, u^{\prime}\right\rangle$ induced by the deletion of $b$ can be defined as follows: first take

$$
A_{i}^{\prime}:= \begin{cases}A_{j} \backslash\{b\} & \text { if } i=j \\ A_{i} & \text { else }\end{cases}
$$

and then define $u_{i}^{\prime}$ as the restriction of $u_{i}$ to $A^{\prime}:=\prod_{i} A_{i}^{\prime}$.

Thus we can give a formal definition of a reduced form of, as follows. First check whether there is a pure strategy of some player that is payoff-equivalent with some other strategy. If there is no such strategy, is called reduced. If there are such strategies, pick one and delete it. This yields a game , ' as previously described. Repeat the process using, ' instead of , etc., until finally (after a finite number of steps) a reduced game results. Such a game is called a reduced form of, . The question now is in what sense reduced forms of, are equal to each other. In order to give a precise meaning to this sense, and to get a short proof, we need another way to represent the deletion of a pure strategy, namely by means of so-called reduction maps. This representation was introduced by Mertens (1987) and can also be found in van Damme (1994).

A game,$^{\prime}=\langle B, v\rangle$ is called a reduction of the game, $=\langle A, u\rangle$ if there is a map $f=\left(f_{i}\right)_{i \in N}$ from $\Delta_{A}$ to $\Delta_{B}$ such that for every $i \in N$

(1) $f_{i}: \Delta\left(A_{i}\right) \rightarrow \Delta\left(B_{i}\right)$ is affine and onto

(2) $u_{i}=v_{i} \circ f$.

The function $v_{i} \circ f$ denotes the composition of $v_{i}$ and $f$. In this situation $f$ is called a reduction map from, to , '. Note that each $f_{i}$ preserves payoff equivalence, i.e., for all $x_{i}$ and $y_{i}$ in $\Delta\left(A_{i}\right)$, $x_{i}$ is payoff equivalent with $y_{i}$ if and only if $f_{i}\left(x_{i}\right)$ is payoff equivalent with $f_{i}\left(y_{i}\right)$.

Roughly speaking, reducing a game captures both the idea of deletion of a pure strategy and the 'relabeling' of strategies. This specific combination makes it an ideal tool to tackle the problem at hand. However, first we need to establish the connection between the deletion of a single pure strategy and reducing a game.

Lemma 1. Let, ' be the game induced by the deletion of a pure strategy $b$ of player $j$ payoff equivalent with some other strategy $z_{j} \in \Delta\left(A_{j}\right)$. Then ,' is a reduction of , .

Proof. We have to show that there is a reduction map $f=\left(f_{i}\right)_{i \in N}$ from , to , '. Obviously for $i \neq j$ we can choose $f_{i}$ to be the identity $i d_{i}: \Delta\left(A_{i}\right) \rightarrow \Delta\left(A_{i}\right)$. For $j$, we define $f_{j}: \Delta\left(A_{j}\right) \rightarrow \Delta\left(A_{j}^{\prime}\right)$ as follows. For $x_{j} \in \Delta\left(A_{j}\right)$ and $a \in A_{j}^{\prime}$,

$$
f_{j}\left(x_{j}\right)_{a}:=x_{j a}+z_{j a}\left(1-z_{j b}\right)^{-1} x_{j b} .
$$


Note that this definition makes sense, since the assumption that $z_{j} \neq b$ implies that $1-z_{j b}$ is larger than zero. It is straightforward to check that $f_{j}\left(x_{j}\right) \in \Delta\left(A_{j}^{\prime}\right)$ and that $f_{j}$ is affine and onto. The fact that $u_{i}=u_{i}^{\prime} \circ f$ can be seen as follows. First note that both $u_{i}$ and $u_{i}^{\prime} \circ f$ are multi-affine maps on $\Delta_{A}$. So we only have to prove that they coincide on the set $A$ of pure strategy profiles. Now note that, for each $a \in A$ with $a_{j} \neq b$, we have that $f_{j}\left(a_{j}\right)=a_{j}$. Furthermore, $u_{i}^{\prime}$ is the restriction of $u_{i}$ to the set $A^{\prime}$ of pure strategy profiles $a \in A$ with $a_{j} \neq b$. So in this case it is clear that $u_{i}(a)=\left(u_{i}^{\prime} \circ f\right)(a)$. Now take a pure strategy profile $c \in A$ with $c_{j}=b$. Then for each $i \in N$,

$$
\begin{aligned}
\left(u_{i}^{\prime} \circ f\right)(c) & =\sum_{a \in A^{\prime}} \prod_{h} f_{h}\left(c_{h}\right)_{a_{h}} u_{i}^{\prime}(a)=\sum_{a_{j} \in A_{j}^{\prime}} z_{j a_{j}}\left(1-z_{j b}\right)^{-1} u_{i}\left(c_{-j} \mid a_{j}\right) \\
& =\left(1-z_{j b}\right)^{-1}\left\{u_{i}\left(c_{-j} \mid z_{j}\right)-z_{j b} u_{i}\left(c_{-j} \mid b\right)\right\} \\
& =\left(1-z_{j b}\right)^{-1}\left\{u_{i}\left(c_{-j} \mid b\right)-z_{j b} u_{i}\left(c_{-j} \mid b\right)\right\}=u_{i}(c) .
\end{aligned}
$$

The penultimate equality follows from the fact that $b$ and $z_{j}$ are payoff-equivalent.

Now we can also capture successive deletions of pure strategies in terms of reduction maps. Suppose that a map $f$ is a reduction map from a game, to a game, ' and that $g$ is a reduction map from ,' to , ". Then it is easy to check that the composition $g \circ f$ of $f$ and $g$ is a reduction map from, to , ". Thus it follows from the previous Lemma that any game, ' obtained from, by the successive (not necessarily exhaustive) deletion of pure strategies is a reduction of ,. So, if we have a way to identify two reduced games that are both reductions of, , we also have a way to identify two reduced forms of , .

\section{Uniqueness of the reduced form}

After thus having translated the process of deletion of strategies in terms of reductions of, , we can again use reduction maps to describe in what way two reduced forms of, are identical.

Two games,${ }^{*}=\langle B, v\rangle$ and,${ }^{* *}=\langle C, w\rangle$ are called isomorphic if there is a reduction map $f$ from ,${ }^{*}$ to,${ }^{* *}$ that is also one-to-one. It is equivalent to require that each $f_{i}$ induces a one-to-one and onto function between $B_{i}$ and $C_{i}$. The well-known phrase 'the reduced form is determined up to the relabeling of pure strategies' refers to the latter property of isomorphic games.

In the proof of the isomorphy of two reduced forms of, we will need the following well-known lemma. For a proof we refer to Lemma 1 of Vermeulen and Jansen (1996).

Lemma 2. Let $f$ be an affine and onto map from a polytope $P$ to a polytope $Q$. Then $\operatorname{ext}(Q)$ is a subset of $f(\operatorname{ext}(P))$.

Now suppose that $f$ is a reduction map from, to , ${ }^{*}$ and that $g$ is a reduction map from, to , ${ }^{* *}$.

Theorem 1. If both, ${ }^{*}$ and, ${ }^{* *}$ are reduced forms of, then, ${ }^{*}$ and, ${ }^{* *}$ are isomorphic. 
Proof. (a) In this part we will only use the fact that,${ }^{* *}$ is a reduced form of, . We will first construct a reduction map $h$ from,${ }^{*}$ to,${ }^{* *}$. To this end, note that for a player $i, f_{i}$ is an affine onto map from $\Delta\left(A_{i}\right)$ to $\Delta\left(B_{i}\right)$. So $B_{i} \subset f_{i}\left(A_{i}\right)$ by the previous Lemma. Then there must exist a map $s_{i}: B_{i} \rightarrow A_{i}$ with $\left(f_{i} \circ s_{i}\right)(b)=b$ for all $b \in B_{i}$. Let $t_{i}: \Delta\left(B_{i}\right) \rightarrow \Delta\left(A_{i}\right)$ be the affine extension of $s_{i}$. Then it is easy to check that $f_{i} \circ t_{i}$ equals the identity $i d_{i}$ on $\Delta\left(B_{i}\right)$. So, if we write $t:=\left(t_{i}\right)_{i \in N}$, then $f \circ t$ equals the identity on $\Delta_{B}$.

Now define $h: \Delta_{B} \rightarrow \Delta_{A}$ as $h:=\left(h_{i}\right)_{i \in N}$ with $h_{i}:=g_{i}$ ot $t_{i}$. Clearly, $h_{i}$ is an affine map. Furthermore, for all $i \in N$ and $y \in \Delta_{B}$,

$$
v_{i}(y)=v_{i}((f \circ t)(y))=\left(v_{i} \circ f \circ t\right)(y)=\left(u_{i} \circ t\right)(y)=\left(w_{i} \circ g \circ t\right)(y)=\left(w_{i} \circ h\right)(y)
$$

because $f \circ t$ is the identity and $v_{i} \circ f=u_{i}=w_{i} \circ g$. So we only need to check that $h_{i}$ is onto. To this end, take a pure strategy $c \in C_{i}$. Again by the previous Lemma we know that there exists a pure strategy $a \in A_{i}$ with $g_{i}(a)=c$. Write $x_{i}:=\left(t_{i} \circ f_{i}\right)(a) \in t_{i}\left(\Delta\left(B_{i}\right)\right)$. Then

$$
f_{i}\left(x_{i}\right)=\left(f_{i} \circ t_{i} \circ f_{i}\right)(a)=\left(i d_{i} \circ f_{i}\right)(a)=f_{i}(a) .
$$

So, since the strategies $f_{i}\left(x_{i}\right)$ and $f_{i}(a)$ are identical, they are certainly payoff equivalent. Then $x_{i}$ and $a$ must also be payoff equivalent, since $f_{i}$ preserves payoff equivalence. Thus, since $g_{i}$ also preserves payoff equivalence, $g_{i}\left(x_{i}\right)$ and $g_{i}(a)=c$ are payoff equivalent. Hence, $c$ and

$$
\left(h_{i} \circ f_{i}\right)(a)=\left(g_{i} \circ t_{i} \circ f_{i}\right)(a)=g_{i}\left(x_{i}\right)
$$

must also be payoff equivalent. However, since, ${ }^{* *}$ is a reduced from of , it is certainly a reduced game. So, since $c$ is a pure strategy, we get that $\left(h_{i} \circ f_{i}\right)(a)=c$. Hence, $C_{i} \subset h_{i}\left(\Delta\left(A_{i}\right)\right)$ and $h_{i}$ must be onto since it is affine.

(b) Now since both, ${ }^{*}$ and , ${ }^{* *}$ are reduced forms of , , part (a) yields a reduction map $h$ from, * to , ${ }^{* *}$ and a reduction map $h^{\prime}$ from , ${ }^{* *}$ to,${ }^{*}$. It is sufficient to prove that the onto map $h$ is also one-to-one. By Lemma 2 we know that both $C_{i} \subset h_{i}\left(B_{i}\right)$ and $B_{i} \subset h_{i}^{\prime}\left(C_{i}\right)$ hold. This however is only possible if $\left|C_{i}\right|=\left|B_{i}\right|$. Hence, $h_{i}$ must be one-to-one.

Conclusion Thus we can interpret the reduced form of a game, as follows. First note that any two reduced games obtained from, by the exhaustive successive deletion of pure strategies are isomorphic, and isomorphy induces an equivalence relation on the class of all normal form games. So, all reduced games that can be obtained from, by successive deletions are contained in the same equivalence class. Hence, the reduced form of the game, can formally be seen as the equivalence class that contains all such reduced games. Practically speaking, any game in this equivalence class can be called the reduced form of, and then this game is said to be unique up to isomorphisms. Hence, 
Theorem 2. A game, ${ }^{*}$ is the reduced form of, if and only if

$(1),{ }^{*}$ is a reduced game and

$(2),{ }^{*}$ is a reduction of, .

\section{Construction of the reduced form}

In this section we will show that for any game, $=\langle A, u\rangle$ the reduced form , ${ }^{*}$ of, can be obtained directly from the game, by the identification of the strategies within certain payoff-equivalence classes. We will first formally define this game, ${ }^{*}$. To that purpose consider the equivalence classes corresponding to the relation of payoff equivalency in the strategy space $\Delta\left(A_{i}\right)$ of player $i$. Let $\mathcal{E}_{i}$ denote the finite collection of those equivalence classes, say $E_{1}, \ldots, E_{S}$, in $\Delta\left(A_{i}\right)$ that contain some pure strategy in $A_{i}$. Let $\mathcal{F}_{i}$ be the collection of those sets in $\mathcal{E}_{i}$ that are a face of $\Delta\left(A_{i}\right)$ and write $\mathcal{F}:=\prod_{i \in N} \mathcal{F}_{i}$. Then, since for each player $i$ and every $E=\left(E_{h}\right)_{h \in N} \in \mathcal{F}$ the payoff function $u_{i}$ is constant on the subset $\prod_{h} E_{h}$ of $A$, we can define $u_{i}^{*}: \mathcal{F} \rightarrow \mathbb{R}$ by

$$
u_{i}^{*}(E):=u_{i}\left(\prod_{h} E_{h}\right) .
$$

So at least $,{ }^{*}:=\left\langle\mathcal{F}, u^{*}\right\rangle$ is a well-defined object. However, in order to show that,${ }^{*}$ is indeed a game, we need to know that $\mathcal{F}_{i}$ is not empty for each player $i$. In other words, we need to show that at least one of these equivalence classes is such a face. In order to prove this, define

$$
B_{i}:=\left\{a \in A_{i} \mid a \in E_{s} \text { for some } E_{s} \in \mathcal{F}_{i}\right\} .
$$

Furthermore, let for a strategy $x_{i} \in \Delta\left(A_{i}\right)$ of player $i$ its carrier be denoted by $C\left(x_{i}\right)$ and let $E_{s}^{*}$ be the collection of pure strategies contained in the equivalence class $E_{s}$. First we need to show the following lemma.

Lemma 3. If $E_{s}$ is not a face of $\Delta\left(A_{i}\right)$, then there is a strategy $z(s)_{i} \in E_{s}$ with $C\left(z(s)_{i}\right) \subset B_{i}$.

Proof. Suppose that we can prove the following proposition:

for every subset $\mathcal{G}_{i}$ of $\mathcal{E}_{i}$ with $\mathcal{G}_{i} \cap \mathcal{F}_{i}=\phi$ we have: for every $E_{s} \in \mathcal{G}_{i}$ there is a strategy $z(s)_{i} \in E_{s}$ whose carrier has an empty intersection with every $E_{t}^{*}$ for which $E_{t} \in \mathcal{G}_{i}$.

Then this is in particular true for $\mathcal{G}_{i}=\mathcal{E}_{i} \backslash \mathcal{F}_{i}$. Thus, for every $E_{s} \notin \mathcal{F}_{i}$ we get a strategy $z(s)_{i} \in E_{s}$ whose carrier has an empty intersection with every $E_{t}^{*}$ for which $E_{t} \notin \mathcal{F}_{i}$. Which means exactly that $C\left(z(s)_{i}\right)$ is a subset of $B_{i}$.

So, we have to show that the proposition $P(k)$ :

for every subset $\mathcal{G}_{i}$ of $\mathcal{E}_{i}$ with $\left|\mathcal{G}_{i}\right|=k$ and $\mathcal{G}_{i} \cap \mathcal{F}_{i}=\phi$ we have: for every $E_{s} \in \mathcal{G}_{i}$ there is a strategy $z(s)_{i} \in E_{s}$ whose carrier has an empty intersection with every $E_{t}^{*}$ for which $E_{t} \in \mathcal{G}_{i}$ holds for every natural number $k$. We will prove this by induction over $k$. To do this we need the following 
(a) Assume that $E_{s}$ is not a face of $\Delta\left(A_{i}\right)$. Then there is a strategy $y(s)_{i} \in E_{s}$ whose carrier is not contained in $E_{s}^{*}$. Now let $z(s)_{i}$ be the strategy obtained by normalizing the non-zero vector $\sum_{a \notin E_{s}^{*}} y(s)_{i a} a$. Then it is straightforward to check that

$$
z(s)_{i}=\left[\sum_{a \notin E_{s}^{*}} y(s)_{i a}\right]^{-1}\left\{y(s)_{i}-\sum_{a \in E_{s}^{*}} y(s)_{i a} a\right\}
$$

is an affine combination of the payoff-equivalent strategies $y(s)_{i}$ and $a$ with $a \in E_{s}^{*}$. Therefore $z(s)_{i}$ is also payoff equivalent with these strategies and hence $z(s)_{i} \in E_{s}$. Furthermore it is clear by construction that the carrier of $z(s)_{i}$ is a subset of the carrier of $y(s)_{i}$ and that it has an empty intersection with $E_{s}^{*}$.

(b) Now we can show $P(1)$ as follows. Note that $P(1)$ is equivalent with: for every $E_{s} \notin \mathcal{F}_{i}$ there is a strategy $z(s)_{i} \in E_{s}$ whose carrier has an empty intersection with $E_{s}^{*}$. This however is a direct consequence of part (a). So, we only need to prove the induction step. To this end, assume that $P(k)$ is true. We will show $P(k+1)$.

Assume that there is a subset $\mathcal{H}_{i}$ of $\mathcal{E}_{i}$ with $\left|\mathcal{H}_{i}\right|=k+1$ and $\mathcal{H}_{i} \cap \mathcal{F}_{i}$ is empty. Take an $E_{s} \in \mathcal{H}_{i}$. Since $k+1 \geq 2$, we can also take an $E_{r} \in \mathcal{H}_{i}$ with $r \neq s$. Then both $\mathcal{G}_{i}:=\mathcal{H}_{i} \backslash\left\{E_{r}\right\}$ and $\mathcal{G}_{i}^{\prime}:=\mathcal{H}_{i} \backslash\left\{E_{s}\right\}$ satisfy the conditions of $P(k)$. So, there are strategies, let us call them $x(s)_{i} \in E_{s}$ and $x(r)_{i} \in E_{r}$, with

$$
C\left(x(s)_{i}\right) \cap \bigcup_{E_{t} \in \mathcal{G}_{i}} E_{t}^{*}=\phi \quad \text { and } \quad C\left(x(r)_{i}\right) \cap \bigcup_{E_{t} \in \mathcal{G}_{i}^{\prime}} E_{t}^{*}=\phi
$$

If also $C\left(x(s)_{i}\right) \cap E_{r}^{*}$ is the empty set, then it is clear that the carrier of $x(s)_{i}$ has an empty intersection with every $E_{t}^{*}$ for which $E_{t} \in \mathcal{H}_{i}$ and we have a proof of the statement for $k+1$.

So, assume that this is not the case, which implies that $\sum_{a \in E_{r}^{*}} x(s)_{i a}>0$. Define the strategy $y(s)_{i} \in E_{s}$ by

$$
y(s)_{i}:=x(s)_{i}+\sum_{a \in E_{r}^{*}} x(s)_{i a}\left[x(r)_{i}-a\right] .
$$

Now suppose that the carrier of $y(s)_{i}$ is a subset of $E_{s}^{*}$. Since $\sum_{a \in E_{r}^{*}} x(s)_{i a}>0$ by assumption and the carrier of $x(r)_{i}$ has an empty intersection with $E_{r}^{*}$, it follows directly from the definition of $y(s)_{i}$ that the carrier of $x(r)_{i}$ is a subset of the carrier of $y(s)_{i}$. So, the carrier of $x(r)_{i}$ must also be a subset of $E_{s}^{*}$. This would imply that $x(r)_{i}$ is an element of $E_{s}$, which is impossible since $x(r)_{i}$ is an element of $E_{r}$ and $E_{r} \neq E_{s}$.

Thus we know that the carrier of $y(s)_{i} \in E_{s}$ is not contained in $E_{s}^{*}$. So, we can apply the construction described in part (a) to $y(s)_{i}$ to obtain a strategy $z(s)_{i} \in E_{s}$ whose carrier is contained in the carrier of $y(s)_{i}$ and has an empty intersection with $E_{s}^{*}$. Now note that the carrier of $y(s)_{i}$ has an empty intersection with $E_{r}^{*}$ and every $E_{t}^{*}$ with $E_{t} \in \mathcal{G}_{i} \cap \mathcal{G}_{i}^{\prime}=\mathcal{H}_{i} \backslash\left\{E_{s}, E_{r}\right\}$. Hence, the 
carrier of $z(s)_{i} \in E_{s}$ has an empty intersection with every $E_{t}^{*}$ for which $E_{t} \in \mathcal{H}_{i}$. This concludes the proof of the induction step.

Now it is easy to show that $\mathcal{F}_{i}$ is not empty. Suppose that it is empty. Then none of the elements of the non-empty set $\mathcal{E}_{i}$ is a face of $\Delta\left(A_{i}\right)$. So, we can take an $E_{s} \in \mathcal{E}_{i}$ that is not a face of $\Delta\left(A_{i}\right)$ and by the previous Lemma there is a strategy $z(s)_{i} \in E_{s}$ such that

$$
C\left(z(s)_{i}\right) \cap A_{i}=C\left(z(s)_{i}\right) \cap \bigcup_{t \in S} E_{t}^{*}=\phi .
$$

Since this is impossible, we know that $\mathcal{F}_{i}$ is not empty. Hence, ${ }^{*}:=\left\langle\mathcal{F}, u^{*}\right\rangle$ is indeed a game.

Finally we will show that, ${ }^{*}$ is indeed the reduced form of , . So, by Theorem 2 , we need to prove that , * is a reduction of, and that , ${ }^{*}$ is a reduced game. First we will prove that , ${ }^{*}$ is a reduction of, . It is convenient to split this proof into two parts. Consider the game,$^{\prime}=\left\langle B, u^{\prime}\right\rangle$ wherein $u_{i}^{\prime}: \rightarrow \mathbb{R}$ is the restriction of $u_{i}$ to the subset $B:=\prod_{i \in N} B_{i}$ of $A$.

Lemma 4. The game, ' is a reduction of, .

Proof. We will show that there exists a reduction map $f$ from, to , '.

To this end, take a player $i$ and a pure strategy $a \notin B_{i}$. Then the equivalence class in $\mathcal{E}_{i}$ that contains $a$ is not an element of $\mathcal{F}_{i}$. So we can use Lemma 3 and choose a strategy $z(a)_{i}$ that is payoff equivalent with $a$ while $C\left(z(a)_{i}\right) \subset B_{i}$. (Obviously we can coordinate these choices in such a way that $z(a)_{i}=z(b)_{i}$ whenever $a$ is payoff equivalent with $b$, but this is not necessary for our argument.) We introduce the map $f_{i}: \Delta\left(A_{i}\right) \rightarrow \Delta\left(B_{i}\right)$ with, for every $x_{i} \in \Delta\left(A_{i}\right)$ and every $b \in B_{i}$,

$$
f_{i}\left(x_{i}\right)_{b}:=x_{i b}+\sum_{a \notin B_{i}} x_{i a} z(a)_{i b}
$$

Note that $f_{i}\left(x_{i}\right)$ is an element of $\Delta\left(B_{i}\right)$ because of the fact that $C\left(z(a)_{i}\right) \subset B_{i}$ for every $z(a)_{i}$. Furthermore, it is easily verified that $f_{i}$ is affine and onto.

So, we only have to show that $u_{i}=u_{i}^{\prime} \circ f$. The exact proof of this, although not difficult, is a bit messy. Therefore we will only present one step of the proof. Take $i, j \in N$ and $x \in \Delta_{A}$. For $b=\left(b_{i}\right)_{i \in N} \in B$, write $b_{-i}=\left(b_{j}\right)_{j \neq i}$ and $\prod_{b_{-i}}=\prod_{h \neq i} f_{h}\left(x_{h}\right)_{b_{h}}$. Then

$$
\left(u_{j}^{\prime} \circ f\right)(x)=\sum_{b \in B} f_{i}\left(x_{i}\right)_{b_{i}} \prod_{b_{-i}} u_{j}^{\prime}(b)=\sum_{b_{-i} \in B_{-i}} \prod_{b_{-i}} \sum_{b_{i} \in B_{i}} f_{i}\left(x_{i}\right)_{b_{i}} u_{j}\left(b_{-i} \mid b_{i}\right) .
$$

Now take a fixed $b_{-i} \in B_{-i}$. Then we can compute that

$$
\begin{aligned}
\sum_{b_{i} \in B_{i}} f_{i}\left(x_{i}\right)_{b_{i}} u_{j}\left(b_{-i} \mid b_{i}\right)=\sum_{b_{i} \in B_{i}}\left[x_{i b_{i}}+\sum_{a \notin B_{i}} x_{i a} z(a)_{i b_{i}}\right] u_{j}\left(b_{-i} \mid b_{i}\right) \\
=\sum_{b_{i} \in B_{i}} x_{i b_{i}} u_{j}\left(b_{-i} \mid b_{i}\right)+\sum_{a \notin B_{i}} x_{i a} \sum_{b_{i} \in B_{i}} z(a)_{i b_{i}} u_{j}\left(b_{-i} \mid b_{i}\right) \\
=\sum_{a \in B_{i}} x_{i a} u_{j}\left(b_{-i} \mid a\right)+\sum_{a \notin B_{i}} x_{i a} u_{j}\left(b_{-i} \mid z(a)_{i}\right) \\
=\sum_{a \in B_{i}} x_{i a} u_{j}\left(b_{-i} \mid a\right)+\sum_{a \notin B_{i}} x_{i a} u_{j}\left(b_{-i} \mid a\right)=u_{j}\left(b_{-i} \mid x_{i}\right) .
\end{aligned}
$$


The third equality follows from the fact that the carrier of $z(a)_{i}$ is contained in $B_{i}$ and the fourth one from the payoff equivalence of $a$ and $z(a)_{i}$. Now the substitution of the result of the second displayed computation into the first one yields

$$
\left(u_{j}^{\prime} \circ f\right)(x)=\sum_{b \in B} \prod_{b_{-i}} u_{j}\left(b_{-i} \mid x_{i}\right)
$$

Thus, repitition of this computation eventually yields the equality $\left(u_{j}^{\prime} \circ f\right)(x)=u_{j}(x)$. Hence, $f$ is a reduction map from, to , '.

Secondly,

Lemma 5. The game, ${ }^{*}$ is a reduction of the game, '.

Proof. Define for player $i$ the map $\pi_{i}: \Delta\left(B_{i}\right) \rightarrow \Delta\left(\mathcal{F}_{i}\right)$ by, for $y_{i} \in \Delta\left(B_{i}\right)$ and $E \in \mathcal{F}_{i}$,

$$
\pi_{i}\left(y_{i}\right)_{E}:=\sum_{a \in E} y_{i a}
$$

and $\pi:=\left(\pi_{i}\right)_{i \in N}$. It is to be shown that $\pi$ is a reduction map from , ' to , ${ }^{*}$. Evidently $\pi_{i}$ is an affine map onto $\Delta\left(\mathcal{F}_{i}\right)$ for every player $i$. So it remains to be shown that $\pi$ preserves payoffs. However, there is a simple argument why $u_{j}^{\prime}=u_{j}^{*} \circ \pi$. Take a pure strategy profile $\left(b_{i}\right)_{i \in N} \in B$. Let $E\left(b_{i}\right) \in \mathcal{F}_{i}$ denote the unique equivalence class that contains $b_{i}$. Then

$$
u_{j}^{*}\left(\pi_{i}\left(b_{i}\right)_{i \in N}\right)=u_{j}^{*}\left(E\left(b_{i}\right)_{i \in N}\right)=u_{j}\left(\prod_{i} E\left(b_{i}\right)\right)=u_{j}\left(\left(b_{i}\right)_{i \in N}\right)=u_{j}^{\prime}\left(\left(b_{i}\right)_{i \in N}\right) .
$$

So, both $u_{j}^{*} \circ \pi$ and $u_{j}^{\prime}$ are multi-affine maps from $\Delta_{B}$ to $\Delta_{\mathcal{F}}$ that agree on the set of extreme points of $\Delta_{B}$. Then they are necessarily identical, which completes the proof.

The last two Lemmas together show that $\pi \circ f$ is a reduction map from , to , ${ }^{*}$. So,,${ }^{*}$ is a reduction of, and, by Theorem 2 , the only thing left to show is

Theorem 3.,${ }^{*}$ is a reduced game.

Proof. Suppose that it is not. Then for some player $j$ there must be a pure strategy $E \in \mathcal{F}_{j}$ that is payoff equivalent with a strategy $z_{j} \in \Delta\left(\mathcal{F}_{j}\right)$, while $z_{j} \neq E$.

Furthermore, since $\pi \circ f$ is a reduction map from , to , ${ }^{*}$ it is certainly onto. In particular, there is a strategy $x_{j} \in \Delta\left(A_{j}\right)$ with $\left(\pi_{j} \circ f_{j}\right)\left(x_{j}\right)=z_{j}$. It is also easily checked that $\left(\pi_{j} \circ f_{j}\right)(b)=E$ given a pure strategy $b \in E$. Then the fact that $\pi_{j} \circ f_{j}$ preserves payoff equivalence implies that $x_{j}$ is payoff equivalent with $b \in E$. So, $x_{j}$ must also be an element of $E$. However, $E$ itself is an element of $\mathcal{F}_{j}$, which means that $E$ is a face of $\Delta\left(A_{j}\right)$. Therefore, $x_{j}$ must be a convex combination of the pure strategies $b \in E^{*}$ in $E$. Since for all these strategies $b \in E^{*}$ it can easily be checked that $\left(\pi_{j} \circ f_{j}\right)(b)=E$, it follows from the affinity of $\pi_{j} \circ f_{j}$ that also $\left(\pi_{j} \circ f_{j}\right)\left(x_{j}\right)=E$. This however contradicts the assumption that $\left(\pi_{j} \circ f_{j}\right)\left(x_{j}\right)=z_{j} \neq E$. Hence,,${ }^{*}$ must be a reduced game. $\triangleleft$ 


\section{Computational aspects}

The actual computation of the reduced form,${ }^{*}=\left\langle\mathcal{F}, u^{*}\right\rangle$ of the game,$=\langle A, u\rangle$ can be done in finite time. To see this we will first argue that for each player $i$ the set $\mathcal{F}_{i}$ can be computed in finite time. To this end, take a pure strategy $b \in A_{i}$ and let $E_{s} \in \mathcal{E}_{i}$ be the unique equivalence class that contains $b$. Write $A_{-i}:=\prod_{h \neq i} A_{h}$. Then $E_{s}$ is exactly the set of points $x \in \mathbb{R}^{A_{i}}$ that satisfy the finite system of linear (in)equalities

for all $j \in N$ and for all $a_{-i} \in A_{-i}: u_{j}\left(a_{-i} \mid x_{i}\right)=u_{j}\left(a_{-i} \mid b\right)$

for all $a \in A_{i}: \quad x_{i a} \geq 0$

and: $\quad \sum_{a \in A_{i}} x_{i a}=1$.

Thus we have a polyhedral description of the polytope $E_{s}$ and the set $\operatorname{ext}\left(E_{s}\right)$ of extreme points of $E_{s}$ can be calculated in finite time. Now note that

$$
E_{s} \in \mathcal{F}_{i} \quad \text { if and only if } \operatorname{ext}\left(E_{s}\right) \subset A_{i}
$$

and that the second condition can also be checked in finite time. So, since there are only finitely many elements $b \in A_{i}$ and every element of $\mathcal{E}_{i}$ occurs at least once in the above procedure when $b$ ranges through $A_{i}$, we have a method to check within finite time exactly which elements of $\mathcal{E}_{i}$ are also elements of $\mathcal{F}_{i}$. Now select exactly one element in each set $\operatorname{ext}\left(E_{s}\right)$ for which $E_{s} \in \mathcal{F}_{i}$. This selection yields a subset $C_{i}$ of $A_{i}$. Write $C:=\prod_{i} C_{i}$ and let $v_{i}$ be the restriction of $u_{i}$ to $C$. Since both the selection process and the evaluation of $u_{i}$ on $C$ can also be done in finite time, we can construct the game,$^{\prime}=\langle C, v\rangle$ from , in finite time. Finally note that,${ }^{\prime}$ and,${ }^{*}$ are isomorphic. Hence,,${ }^{\prime}$ is the reduced form of, and can be derived from, in a finite number of steps.

Example For the strategy space $\Delta_{4}$ of the second player of the $2 \times 4$-bimatrix game

$$
=\left[\begin{array}{llllll}
0, & 1 & 0, & 2 & 0,-1 & 0,0 \\
0,-1 & 0,-2 & 0, & 1 & 0,0
\end{array}\right]
$$

there are 4 equivalence classes containing a pure strategy:

$$
\begin{aligned}
& E_{1}=\operatorname{ch}\left\{e_{1}, \frac{2}{3} e_{2}+\frac{1}{3} e_{3}, \frac{1}{2} e_{2}+\frac{1}{2} e_{4}\right\} \\
& E_{2}=\left\{e_{2}\right\} \\
& E_{3}=\left\{e_{3}\right\} \\
& E_{4}=\operatorname{ch}\left\{e_{4}, \frac{1}{3} e_{2}+\frac{2}{3} e_{3}, \frac{1}{2} e_{1}+\frac{1}{2} e_{3}\right\} .
\end{aligned}
$$

Clearly $\mathcal{F}_{2}=\left\{E_{2}, E_{3}\right\}$. Hence, the reduced game of , is the game $\left[\begin{array}{lll}0, & 2 & 0,-1 \\ 0,-2 & 0, & 1\end{array}\right]$ obtained by 
deleting the first and last column.

\section{References}

1. van Damme, E.E.C. (1994) Strategic Equilibrium, in: Handbook of Game Theory with Economic Applications, Vol. 3. Elsevier. (preliminary draft)

2. Kohlberg, E. and Mertens, J.F. (1986). On strategic stability of equilibria, Econometrica 54, $1003-1037$.

3. Mertens, J.F. (1987) Ordinality in noncooperative games, Core Discussion Paper 8728, CORE Louvain de la Neuve, Belgium.

4. Vermeulen, A.J. and M.J.M. Jansen (1996) Extending invariant solutions, Games and Economic Behaviour (forthcoming). 